\title{
VOM FETISCH BIS ZUM DRAMA? ANMERKUNGEN ZUR RENAISSANCE DER KULTURWISSENSCHAFTEN
}

\begin{abstract}
Die Verwirrung des Sprachgebrauchs steigert sich, je mehr der Begriff Kultur zu einer Modesache wird; ja man kann sagen: je mehr die Wissenschaft sich des Begriffs annimmt.

Fritz Mauthner
\end{abstract}

Zweierlei fordert zum Nachdenken heraus. Erstens: Warum wollen oder sollen die Literaturdisziplinen den altehrwürdigen Rock der Geisteswissenschaften abstreifen? Zweitens: Welche neue Anmut, welchen Zugewinn verspricht ihnen das modische Kleid der Kulturwissenschaften? Es ist gewiß nicht nur eine Frage des Kostümwechsels, der die aktuellen Debatten erregt. ${ }^{1}$ Eine so nominalistische Einstellung könnte man getrost sich selbst überlassen. Die Begriffe "Kultur" und "Geist" sind eben Begriffe und nicht nur Worte, und was sie ergreifen oder bezeichnen, das ist kategorisch geschieden, auch wenn sie auf den ersten Blick als Gemeinsames nur die banalen Eigenschaften eines Vater-Kind-Verhältnisses preiszugeben scheinen.

\footnotetext{
1 Vgl. folgende Sammelbände: Hendrik Birus, ed., Germanistik und Komparatistik (Stuttgart: Metzler, 1995); Renate Glaser und Matthias Luserke, ed., Literatumissenschaft - Kultumvissenschaft: Positionen, Themen, Perspektiven (Opladen: Westdeutscher Verlag, 1996); Lutz Danneberg und Friedrich Vollhardt, ed., Wie international ist die Literaturwissenschaft? Methoden-und Theoriediskussion in den Literatumissenschaften: Kulturelle Besonderheiten und interkultureller Austausch am Beispiel des Interpretationsproblems (1950-1990) (Stuttgart: Metzler, 1996); Hartmut Böhme und Klaus Scherpe, ed., Literatur und Kulturwissenschaften: Positionen, Theorien, Modelle (Reinbek: Rowohlt, 1996).
} 
Noch ist es nicht lange her, da galt Kultur als Geistschöpfung. Wer aus Profession - ob Philologe, Historiker oder Philosoph - über Kultur nachdachte oder forschte, verstand sich daher mit Blick auf die prima causa als Geisteswissenschaftler. Und das hieß viel. Denn am "Geist" als Subjekt jeglicher Sinnstiftung teilzuhaben, war als Legitimation für die wissenschaftliche Arbeit am Sinn nicht zu überbieten. Von heute aus gesehen erscheinen diese wissenschaftlichen Bemühungen um den "Geist" der Kultur als Kennzeichen einer sozialen Funktion, die den beschleunigten Ausdifferenzierungsproze $\beta$ konfligierender kultureller Felder in der Moderne auf ein einheitstiftendes Prinzip zurückzuführen suchte.

"Von heute aus..." - was heißt das hier und jetzt für die Kultur, den Geist, den Sinn? Eine Bibliothek von Antworten gibt es auf die Frage, die sich jedoch - was die Einstellungen gegenüber den westlichen Gesellschaften betrifft - auf eine spannungsreiche, die Gegenwart charakterisierende Ambiguität reduzieren lassen: Die Sphären fallen auseinander und treten zugleich in ein Austauschverhältnis. Denn einerseits soll Kultur - folgt man Daniel Bells Diagnose in The Cultural Contradictions of Capitalism (1976) - zum Konsumstil, Sinn zur Beliebigkeit verkommen sein; Hintergrund für diese Kritik ist ein Dennoch, da Bell mit einem quasi-religiösen, integrativ wirkenden Kultwert gegen den dissoziierenden Gebrauchsund Ausstellungswert der Oberflächenkultur zu Felde zieht und mit dem Ruf nach moral guidance ein liberalistisches Credo kohärenzstiftender Sinnfindung beschwört. Andererseits soll "Kultur" heute - folgt man der Diagnose von Panajotis Kondylis in Niedergang der bürgerlichen Denk- und Lebensformen (1991) - für eine massendemokratische Erscheinung stehen, deren Egalitätsprinzip sich über die traditionelle Unterscheidung zwischen 'Hoch'- und Populärkultur hinwegsetzt, um die Wertdifferenzen zwischen beiden Sphären in einem osmotischen Austauschprozeß auszugleichen. Zirkulation, Rekursivität, Fluktuation, Dissipation usf. lauten die neuen Begriffsgitter, durch die wie durch alchemisch aktive Filter das moderne, synkretistisch fortwuchernde Gespinst der Kulturen hindurch muß, damit der sozial- und kulturwissenschaftliche Blick in den Stand gesetzt wird, lesbare Muster unterscheiden zu kön- 
nen. " "Kultur", zum formellen Arbeitsbegriff der Kultur-Wissenschaften transformiert, kommt ohne Geistsubstanz aus. Die Entscheidung ist pragmatisch und wissenschaftsrational. Denn der formelle Begriff erleichtert und stärkt die Ermittlung positiven Wissens, und es stellt sich die Frage, ob das auf Kosten jener Reflexion geschieht, für die der Geist-Begriff steht. Von Berlin bis Passau hat die Kulturwissenschaft bereits ein eigens so genanntes Berufsstudium in die akademische Welt gesetzt. Etwas ähnliches von der Geisteswissenschaft zu verlangen, würde nur Spott verdienen, da diese Bezeichnung seit Diltheys Tagen als Sammelcode für jene Fakultäten in Gebrauch ist, die sich nicht der Naturerkenntnis, sondern - wie es bei dem Wissenschaftsphilosophen heißt - der Konstruktion der "geschichtlich-gesellschaftlichen Wirklichkeit" widmen. ${ }^{3}$ Hier werden Wirklichkeit und Geist in ein Verhältnis gesetzt, das mehr umfaßt als das in materielle und ideelle Güter aufgeteilte Reich der Kultur. Was zu Diltheys Zeiten Konstruktion des Vergangenen hieß, stand im Dienst einer Krisentherapie, die von der autonomiestärkenden Kraft der nationalen Bildungs-Erlebnisse noch überzeugt sein mochte. Den supra- und transnationalen Kräften, die heute das klassische Modell des Nationalstaates unter-

2 Vgl. den gehaltvollen Artikel "Kultur als System" von Walter F. Bühl, Kultur und Gesellschaft, ed. F. Neidhardt et al. Sonderheft 27 der Kölner Zeitschrift für Soziologie und Sozialpsychologie (Opladen: Westdeutscher Verlag, 1986) 118-44.

3 Dilthey verwendet den Konstruktionsbegriff mit unterschiedlicher Gewichtung. Er gilt dort, wo es um die "ganze geschichtlich-gesellschaftliche Wirklichkeit" geht, da diese auf einer zufallsbedingten Überlieferung beruht, deren Lücken nur konstruierend zu schließen sind. Er ist dort unangebracht, wo es um die Beziehungen zwischen Teil und Ganzem geht, das sich nur dem Verstehen erschließt. Vgl. Gesammelte Schriften, Bd. I: Einleitung in die Geisteswissenschaften. 1883 (Leipzig: Teubner, 1922) 21 u. 31. In der späteren, 1907-1910 unter dem Eindruck der Phänomenologie entstandenen Schrift Der Aufbau der geschichtlichen Welt in den Geisteswissenschaften, Gesammelte Schriften 7, 5. Aufl. (Göttingen: Vandenhoeck \& Ruprecht, ${ }^{5} 1968$ ) wird der Begriff (nota bene das Äquivalent "Aufbau") der theoretischen Grundlegung und der idealtypischen Bestimmung der Forschungsgegenstände vorbehalten. - Die Klammern im Anschluß an die Dilthey-Zitate im Text verweisen auf Band und Seite der Gesammelten Schriften. 
wandern und delegitimieren, entspricht auf ästhetischer Ebene die Kreolisierung der Kulturen. ${ }^{4}$

Um der Unterscheidung, ja den Widersprüchen zwischen Kultur- und Geisteswissenschaften auf den Grund zu gehen, lohnt es sich, bei dem nachzuschlagen, dessen Name zum Inbegriff für das geworden ist, was heute zur Disposition steht. Überdies zieht dieser Name sich wie ein roter Faden sogar durch die Diskurse der transatlantischen Grundlagentexte der Literary \& Cultural Studies - von Wellek-Warren bis Clifford Geertz und Victor Turner, jenen Ideengebern für eine ethnologisch inspirierte Poetics of Culture; und die Literaturwissenschaften Rußlands, lese ich zu meiner Verwunderung in einem Bericht über deren aktuellen Stand, sollen sich von der DiltheyZeit überhaupt noch nicht losgesagt haben. ${ }^{5}$ Dilthey selbst verdankte übrigens die Bezeichnung "Geisteswissenschaft", die gern als eine idealistische Wortschöpfung angesehen wird, der Übersetzung der englischen Begriffsfügung "moral science" aus John Stuart Mills System of Logic von 1843; eine Übersetzung, die sich zum Original noch unentschieden verhielt, da sie den Ausdruck "Geisteswissenschaften" der wörtlichen Übertragung "moralische Wissenschaften" als Explicans - wie zu vermuten ist - hinzugefügt hat. ${ }^{6}$

Der Fall erscheint mir charakteristisch für jene Translatio-

4 Zur Positionierung der neuen Weltliteratur (world fiction) zwischen den Kulturen vgl. meinen Essay "Nomadisierende Schreibweisen und Lesarten des Fremden”, Neue Rundschau 105 (1994): $52 \mathrm{ff}$.

5 Vgl. Alexander Michailow, "Interpretieren und Verstehen vor dem Erfahrungshintergrund der russischen Literaturwissenschaft", Danneberg/Vollhardt 381 (vgl. Anm. 1). Geertz beruft sich für die Rechtfertigung des hermeneutischen Zirkels in der Ethnographie auf Dilthey, Turner darüber hinaus auf dessen Konzepte der "Weltanschauung" und der "gelebten Erfahrung"; C. Geertz, Local Knowledge: Further Essays in Interpretive Anthropology (New York: Basic Books, 1983) 69; V. Turner, The Anthropology of Performance, 2nd ed. (New York: PAJ, 1992) 84, 95 ff. u. ö. - Zur Bezugnahme der Poetics of Culture auf die Kulturanthropologie vgl. den von H. Aram Veeser herausgegebenen Sammelband The New Historicism (New York: Routledge, 1989), hier vor allem die Beiträge von Greenblatt, Montrose und Pecora.

6 Erich Rothacker, Logik und Systematik der Geisteswissenschaften (Bonn: Bouvier, 1947) $4 \mathrm{ff}$. 
nen im grenzüberschreitenden Kommerz der Wissenschaften, die sich in den gegenwärtigen Diskussionen über das Woher und Wohin der historisch-philologischen Wissenschaften überschlagen. Auch hier gilt indes, was jede Übersetzung auszeichnet: Sie verschiebt die Bedeutungen. Wenn hier und jetzt von "Kulturwissenschaft" die Rede ist, so mag dahinter - sieht man von den volkskundlichen und DDR-Varianten einmal ab - das angloamerikanische Paradigma der "Cultural Studies" zu ahnen sein. Dennoch läßt sich das eine nicht ins andere übersetzen. Die Differenz liegt sicher nicht nur in der Beziehung des einen auf eine Vielfalt von Wissenschaften, des andern auf das, was in den heimischen Philologien unter dem Titel "Landeskunde" versammelt ist. Denn in der angloamerikanischen Wissenschaftskultur stehen die "Cultural Studies" längst für autonome sozialwissenschaftlich-kulturanthropologische Forschungs- und Studienfelder, die allein mit der Elle philologisch-historischer Konventionen nicht auszumessen sind. ${ }^{7}$

Wünschenswert wäre sicher eine Konfrontation zwischen beiden Paradigmen, zumal das geistesgeschichtliche, im deutschen Wissenschaftsbetrieb verwaltete Nebengebäude der Literaturwissenschaften in Auflösung begriffen ist. Der Effizienzdruck der politischen Instanzen, der Verfall eines einst gesicherten Objektbereichs (Kanon) und die Tatsache, daß beruflich nur noch 3\% der Magisterabsolventen philologischer Disziplinen im Bildungssektor unterkommen, sind - um nur einige Ursachen zu erwähnen - der Grund dafür, daß die verschämte Frage "Wozu Literaturwissenschaft?" unverdrossen dauernd neu aufgelegt wird. ${ }^{8}$ Bildung, einst mit einer "Kultur" identisch, die ins offene Meer der Selbstbestimmung münden sollte, liegt in Akademiens Landschaften in einem ausgetrockneten Bett. Der Fluß ist umgelenkt worden: in Kanäle speziellen Kompetenzer-

7 Vgl. G. Turner, British Cultural Studies: An Introduction (Boston: Unwin Hyman, 1990); Lawrence Grossberg et al., ed., Cultural Studies (New York: Routledge, 1992).

8 Martha Meyer-Althoff, "Studium mit Magister-Abschluß", Informationen für die Beratungs- und Vermittlungsstelle der Bundesanstalt für Arbeit 33 (1994). Vgl. ferner: Frank Griesheimer und Alois Prinz, ed., Wozu Literatumwissenschaft? Kritik und Perspektiven (Tübingen: Francke, 1991). 
werbs, zwischen denen es kaum Verbindungen gibt. So scheint es denn an der Zeit, ohne falsche Pietät gegenüber konventionellen Wertstandards neue Perspektiven, Ausbildungsziele und Berufsstudiengänge zu entwickeln, die "Kultur" - in der Vergangenheit meist nur in der engen Bedeutung von Literatur und Künsten verstanden - nicht mehr als etwas Selbstverständliches hinnehmen oder mit Bildung verwechseln. Aufs Äquivalent von Bildung reduziert, verströmt "Kultur" heute etwas Altväterisch-Bürokratisches und fristet ein öffentlich subventioniertes Scheinleben. Als Äquivalent des Marktes indes ist sie längst Stoff für eine gigantische Unterhaltungsindustrie, auf die sich - unter dem Schlagwort "Praxisorientierung" - langsam aber sicher die Zielvorstellungen der Studienreformer einpendeln. ${ }^{9}$

Seit dem Kulturboom der 80er Jahre ist hier manches in Gang gekommen, was dem traditionell ausgebildeten Philologen nicht ganz geheuer erscheint. Meine persönlichen Erfahrungen mit der Studienreform, an der ich vor Ort und qua Amt beteiligt bin, geben mir oft genug das Gefühl, der alltägliche wissenschaftspolitische Pragmatismus verdränge jene Feiertagsgrübelei, die sich nach einer bautechnischen Metapher "Grundlegung" nennt. Die Irritation zu beruhigen - was nicht unbedingt das Beste sein muß - würde wahrscheinlich einen erheblichen Theorieaufwand erfordern, vor dem die Verfallsdaten konventioneller Opiate neu sortiert werden müßten. Ich kann mich im folgenden dem Komplex nur annähern und versuche das in Form von Anmerkungen, die dem Rechnung tragen wollen, was ich den "experimentellen Zustand" nenne und für eine passende Beschreibung des geordneten Durcheinanders halte, das sich Literaturwissenschaft nennt. Mein Versuch gilt daher vorab Unterscheidungen, die den wissenschaftshistorischen und -theoretischen Rahmen der philologischen Disziplinen betreffen. Hier ist das Ziel die Vergegenwärtigung älterer, nicht un-

9 Günter Blamberger et al., ed., Berufsbezogen Studieren: Neue Studiengänge in den Literatur-, Kultur- und Medienwissenschaften (München: Beck, 1993); Georg Jäger und Jörg Schönert, ed., Wissenschaft und Berufspraxis: Angewandtes Wissen und praxisorientierte Studiengänge in den Sprach-, Literatur-, Kultur-und Medienwissenschaften (Paderborn [im Druck]). 
bekannter, aber vielleicht zu unrecht vergessener Problemlagen. Denn das so diffus sich ausbreitende Fahnenwort "Kulturwissenschaft" hat eine Geschichte, deren Studium, soll es genauer bestimmt werden, sich allemal auszahlt. Von einer $R e$ naissance spreche ich daher nicht im Sinne der Wiedergeburt, sondern um jene Komplexitätssteigerung durch Wiederanknüpfen zu bezeichnen, die sich selber als Teil des Kulturwandels verstehen darf. Ein letzter, nur andeutungsweise ausgeführter Schritt wird mich dann zu dem führen, was sich als "Rückkopplungseffekt" bezeichnen läßt, nämlich die Stärkung literaturkritischer Konzepte auf ihrem Weg durch die Gravitationsfelder kulturanalytischer Fragestellungen. Mein Verfahren wählt einen problemgeschichtlichen Fluchtpunkt, die Darstellung bleibt, so abschreckend das klingt, rein theoretisch.

In der 1883 zum erstenmal erschienenen Einleitung in die Geisteswissenschaften rechtfertigt Dilthey - um auf ihn wieder zurückzukommen - seinen epochemachenden Grundlegungsversuch mit einem inner- und einem außerwissenschaftlichen Argument. Zum einen liest er am avancierten Stand der naturwissenschaftlichen Theoriebildung und Methodologie die Gefahr einer unzulässigen Übertragung positivistischer Verfahren auf das Gebiet soziohistorischer Forschungen ab. Zum andern verweist er auf die gesellschaftlichen Umwälzungen seit der Französischen Revolution, um mit Nachdruck hinzuzufügen:

Die Erkenntnis der Kräfte, welche in der Gesellschaft walten, der Ursachen, welche ihre Erschütterungen hervorgebracht haben, der Hilfsmittel eines gesunden Fortschritts, die in ihr vorhanden sind, ist zu einer Lebensfrage für unsere Zivilisation geworden. Daher wächst die Bedeutung der Wissenschaften der Gesellschaft gegenüber denen der Natur; in den großen Dimensionen unseres modernen Lebens vollzieht sich eine Umänderung der wissenschaftlichen Interessen. $(\mathbf{I}, 4)$

An dieser Stelle heißt verkürzt "Wissenschaften der Gesellschaft”, was Dilthey wenige Seiten später programmatisch unter dem Begriff "Geisteswissenschaften" zusammenführt. Er 
zieht die Bezeichnung anderen vor, weil die damals gebräuchlichen - "Gesellschaftswissenschaften (Soziologie), moralische, geschichtliche, Kulturwissenschaften" (I, 6) - ihm zu eng erscheinen. Sie erfassen nicht jene komplexen soziohistorischen Modifikationen, die er mit anthropologischer Emphase auf die "psychophysische Totalität der Menschennatur" bezieht. Das "moderne Leben" ist, so versichert er uns, anders als das vorrevolutionäre beschaffen, nämlich von weitreichenden, Politik, Gesellschaft, Kunst und Denken erfassenden "Umwälzungen" stigmatisiert, deren Erkenntnis zur Überlebensfrage geworden ist. Es sind mithin lebenspraktische Motive, die ihn veranlassen, den Wissenschaften, die sich der "geschichtlich-gesellschaftlichen Wirklichkeit" widmen, einen einheitlichen Zusammenhang zu geben, sie als "ein Ganzes" zu konzipieren, für dessen intellektuellen Führungsanspruch schließlich der Sammelcode "Geisteswissenschaften" einsteht (I, 4). In ihrer idealen 'Ganzheit' bilden die unter diesem Dach versammelten Einzeldisziplinen daher nicht nur ein autonomes und zugleich wissenschaftskritisches Komplement zur Einheit der Naturwissenschaften. Sie antworten vielmehr auch auf die Partikularisierung der lebensweltlichen Erfahrungen in der modernen Zivilisation. Kurz, sie sind in der von Dilthey konzipierten erlebnis- bzw. erfahrungsstimulierenden Funktion das Komplement zur kulturellen Desintegration der modernen Gesellschaft unter der Vorherrschaft des Kalküls; mit einem Wort: Krisenwissenschaften!

Die hier naheliegende Frage, welche Bedeutung der Philosoph dem Terminus "Kultur" in seinem Plan zumißt, ist auf der Ebene der einzelwissenschaftlichen Organisation nicht eindeutig $\mathrm{zu}$ beantworten. Das ist umso bemerkenswerter, da dieser Begriff zu seiner Zeit bereits eine ähnliche Konjunktur erfuhr wie an unserem Fin de siècle. Und auch damals waren es Anthropologie und Ethnologie, deren besondere katalysatorische Effekte Dilthey durchaus anerkannte, die so etwas wie die Tendenz zu einer Universalwissenschaft der Weltkulturen mit unterschiedlicher disziplinärer Gewichtung hervorgerufen haben: Klemms Allgemeine Kulturgeschichte (1842-1853), Spencers kultursoziologische Analysen in Principles of Sociology (1876-96), Graebners in Methoden der Ethnologie (1911) entwickelte Theorie der Kulturkreise usf. 
Diltheys Versuch ist nicht weniger universell ausgerichtet als die genannten Werke, übertrifft diese aber in der Art der theoretischen Konzeptualisierung. Er unterscheidet zwischen drei analytischen Ebenen, auf denen die (1.) "Einzelvölker", (2.) die "äußere Organisation der Gesellschaft" (Staat, Verband etc.) und (3.) die in diese eingekapselten "Systeme der Kultur" (Religion, Recht, Kunst etc.) als wissenschaftlich zu erforschende Objekteinheiten erscheinen (I, 41f.). So abstrakt die wissenschaftlichen Konzepte sind, so methodologisch der zu stiftende Zusammenhang zwischen den Einzeldisziplinen unter dem Dach der "Geisteswissenschaften" ist, Dilthey besteht auf der Erkenntnis des "wirklichen Lebens". Sein pädagogisches Ziel ist, die Nebel der Abstraktion aufzulösen und "diese Wirklichkeit sehen (zu)" lehren (I, 42). Aus diesem Grund gibt er das Modell einer organischen Grundverfassung der Menschheit, in der "Kultur" und "Natur" engste Korrelatbegriffe sind, nie ganz auf. Der analytische Blick sondert zwar zunächst zwischen menschlicher Natur ("psychologischen Tatsachen"), Sozialstruktur und Kultursystemen, hebt die Trennung aber wieder auf, indem er die Organisation der Gesellschaft dem Äußeren, die Gebilde der Kultur dem Inneren zuordnet. Worauf es ankommt, ist Teil jener Wirklichkeits-Anschauung, die den ästhetischen Kern dieser Wissenschaftskonzeption bildet. Die Anschauung kommt dann zum Zug, wenn die zuvor analytisch gesonderten Seiten des Lebens in ihrer organischen Wechselwirkung aufgefaßt werden sollen. Dieser Prozeß besitzt eine Zirkelstruktur, da schon das analytische Studium des Details unter der Präsumtion eines Ganzen geschieht: Der bestimmte Ausdruck, das einzelne Werk oder Symbol wird nicht isoliert, sondern innerhalb eines Verweisungszusammenhangs wahrgenommen, dessen Semantik sich in dem Maße klärt, in dem die Analyse die Elemente und ihre Relationen durchschaut.

Ein Schlüsselbegriff dieser Theorie ist folgerecht der des $Z u$ sammenhangs. In der Perspektive nomothetischer Logik erscheint er als "Kausalzusammenhang", in der geisteswissenschaftlichen als semantisches, auf die innere Kohärenz verweisendes Konstrukt, d.h. als "Bedeutungszusammenhang". Die Lebensformen entfalten sich innerhalb von Zusammenhängen, die der wissenschaftlich-analytische Blick nachträglich ausein- 
anderreißt. Die Vielfalt des Lebens - und die Wechselwirkungen zwischen Sprache, Kultur, Institutionen und semantischen Codes - wieder zusammenzuführen (Synthesis), ist daher nicht Sache des Intellekts, sondern der synthetisch verfahrenden "Einbildungskraft". Sie bindet nicht nur das begrifflich Vereinzelte in eine formelle Einheit, sondern assimiliert es darüber hinaus dem Bedürfnis der Gegenwart und ist daher der entscheidende Faktor im kulturellen Bildungsprozeß.

Im Essay über Novalis - 1865 und wieder 1906 - zitiert Dilthey zustimmend den Satz des Frühromantikers: "Die Welt ist eine sinnlich wahrnehmbare, zur Maschine gewordene Einbildungskraft." ${ }^{10}$ Schon in diesem Aphorismus schlummert eine pansymbolistische Tendenz, die sich zwischen den später entstehenden interpretierenden Einzelwissenschaften zu einem gemeinsamen Kerngebiet verfestigen wird. Die dem MaschinenBild implizite Kritik trifft auch die positiven Wissenschaften, denn über die als unendliche Fülle der endlichen Auslegung entzogene Natur hat kein noch so eindeutiger Begriff Verfügungsgewalt. Die Genealogie der menschlichen Natur läßt sich jedoch an der Entwicklungsgeschichte ihrer kulturellen Objektivationen ablesen, zu denen nicht nur Künste und Literatur (im engeren Sinne der Dichtung), sondern auch jene Gestalten des Geistes gehören, die in den Texten des wissenschaftlich-philosophischen Denkens aufgehoben sind. Was Natur ist, wird daher zuletzt allein jener Anschauung verständlich, die an die Stelle des unzugänglichen Inneren ein "symbolisches Bild" setzt, die Natur - mit einem Wort Diltheys - als "Universaltropus des Geistes" wahrnimmt. Die bekannte hermeneutische Variante dieses Satzes lautet, daß wir die Welt nur "nach Analogie unseres Ich aufzufassen vermögen". ${ }^{11}$ Die in Diltheys Novalis-Lektüre enthaltene Einsicht ist zwingend, löst sie doch die gewöhnliche, zur Definition beider Seiten herangezogene Dichotomie Natur/Kultur auf, um zu verstehen zu geben, daß die Rede von

10 Ich zitiere aus der 14. Auflage von Das Erlebnis und die Dichtung (zuerst 1906) (Göttingen: Vandenhoeck \& Ruprecht, 1965) 212. In einer langen Anmerkung, die Dilthey der 1. Auflage hinzugefügt hat (324f.), bekräftigt er noch einmal die Vorläuferrolle des Novalis für die eigene Wissenschaftsphilosophie.

11 Dilthey, Das Erlebnis 212. 
einer außerhalb der Kultur stehenden Natur keinen Sinn macht. Ob Anschauung, Erkenntnis oder Bearbeitung der Natur, es geht immer um einen symbolisch, d. h. kulturimmanent präformierten Erkenntnis- und Aneignungsmodus.

Wolf Lepenies hat in seiner Untersuchung Die drei Kulturen Dilthey den rebellischen Titel "Partisan der Dichtung in den Bezirken der Wissenschaft" zuerkannt. ${ }^{12}$ Damit trifft er einen entscheidenden Zug. Denn Diltheys Grundlegung der Geisteswissenschaften aus dem Geist der Dichtung läßt sich als Beleg für einen Schub im europäischen Kulturprozeß lesen, dessen Ausläufer bis in die Gegenwart reichen. In diesem Sinne wirft die Ersetzung der positivistischen Begriffslogik durch Symbol und Tropus ein aufschlußreiches Licht nicht nur auf den zur Zeit Diltheys sich vollziehenden Umbau der Kultursysteme. An die Stelle der Philosophie als Architektin der Kultur tritt die Literatur, und das Blatt wendet sich von der Herrschaft der Wahrheit zu der des Diskurses; mit den ironischen Worten eines philosophischen Kommentators: "Everything can be changed by talking in new terms." 13

Dilthey selbst hat ein Jahr vor seinem Tod (1911) in einem groben, Jahrhunderte umfassenden Geschichtsabriß mit Lehrstückcharakter, den er der dritten Auflage des Erlebnis-Buches voranstellte, den "Gang der neueren europäischen Literatur" skizziert. Dieser Abriß liest sich wie eine sonderbare Mixtur aus teleologischen, symbolistischen, dramatologischen und kulturphilosophischen Komponenten. Zum einen rekonstruiert er den Emanzipationsprozeß der Einbildungskraft, deren poetische Gestalt seit dem 18. Jahrhundert in Gegensatz zur Wissenschaft (die ihrerseits nicht völlig der Phantasie entsagt) und zur bürokratischen Rationalisierung in Politik und Gesellschaft tritt. Zum anderen beschreibt er den konfliktreichen Prozeß soziokultureller Differenzierungen in Stadt und Gesellschaft seit der frühen Neuzeit unter den Bildern einer zunehmenden Dramatisierung der Lebensformen. Die Literatur antwortet auf diesen Prozeß, indem sie ihre überkommenen "Strukturen"

12 Wolf Lepenies, Die drei Kulturen: Soziologie zwischen Literatur und Wissenschaft (München: Hanser, 1985) 262.

13 Richard Rorty, Consequences of Pragmatism: Essays 1972-1980 (Minneapolis: U of Minnesota P, 1982) 149. 
und Genres in Schüben reorganisiert, darüber aber das aus dem Blick verliert, was der Autor die "zeitlose Tragik des Menschendaseins" nennt. ${ }^{14}$

Für meine Betrachtung ist diese Skizze deshalb von Bedeutung, weil sie Elemente enthält, die noch in die kultur- bzw. geisteswissenschaftlichen Diskussionen der 20er und 30er Jahre hinüberspielen und nicht zuletzt zum Problemhorizont gegenwärtiger Debatten gehören. Ich fasse hier einige dieser Elemente in aller Kürze zusammen und verlängere hier und da grosso modo - die Reichweite der damit angesprochenen Probleme bis in die Gegenwart:

Erstens: Der Vorstellung vom soziohistorisch beschreibbaren "Drama des Lebens" steht ein erhabener, nämlich tragischer Kulturbegriff gegenüber, der auf Nietzsche zurückweist und sich im Zentrum von Georg Simmels kulturphilosophischen Studien wiederfindet. Die Verschmelzung der Kultur, deren höchste Gestalt im Kunstwerk kristallisiert, mit einer zeitlosen Daseinstragik gilt offenbar der Wiederherstellung ihrer kultischen Bedeutung, deren Zerfall Baudelaire in seinem kleinen Prosapoem "Perte d'auréole" (1865) ins Bild gesetzt, und den Walter Benjamin als Auraverlust beschrieben hat. Dem stocknüchternen, mit Dilthey befreundeten Literarhistoriker Wilhelm Scherer blieb das Umschlagen des Kultwertes in den Marktwert kein Geheimnis: "Die Poesie oder, besser gesagt, das poetische Product", notierte er Ende der 80er Jahre, "ist heute eine Ware wie eine andere." ${ }^{15}$ Eine so zutreffende wie unverschämte Feststellung, die selbst heute noch, auf dem Flügel der konservativen Kulturkritik, Rufe nach einer Re-Auratisierung der Kultur laut werden läßt. Schönsten Ausdruck hat diese Tendenz in George Steiners Essay Real Presences (1989) gefunden, in dem die parasitäre Eintagskultur am mystischen Kern des Kunstwerks zuschanden werden soll.

Dilthey selbst verwendet den Kulturbegriff, soweit ich sehe, eher uneinheitlich; $d$. h. sowohl in analytischer als auch in normativer Funktion: analytisch dort, wo er zwischen Kultursystemen und sozialer Organisation unterschied; normativ dort, wo

14 Dilthey, Das Erlebnis 17.

15 W. Scherer, Poetik, ed. R. M. Meyer (Berlin: Weidmann, 1888) 122. 
er (z. B. im literarhistorischen Vorspann des Erlebnis-Buches) vom Ziel "persönlicher und geschichtlicher Selbstbestimmung" sprach. Die Gegenüberstellung zwischen dem gewöhnlichen Drama des Lebens einerseits und der überhöhten kulturellen Tragik andererseits scheint andeuten zu wollen, daß der Kultwert als kulturelle Norm einen Ort besetzen sollte, der jenseits aller historisch modifizierten, durch Bürokratisierung und Vergesellschaftung verursachten Konflikte und Zerfallserscheinungen liegt. In der aktuellen Diskussion über die Rolle und Funktion der "Geisteswissenschaften" bildet sich, wie mir scheint, eine Tendenz heraus, die Dialektik von Konstanz und Wandel, auf die sich das einst favorisierte Paradigma aufrecht zu erhaltender Kulturwerte bezog, im Rahmen der Historischen Anthropologie zu erörtern. ${ }^{16}$

Zweitens: Eine andere Frage ist die, wie der Objektbereich des Diltheyschen Geisteswissenschaftlers sich näher bestimmen läßt. Wenn die interpretierenden Wissenschaften Teil einer Kultur sind, deren Ziel "Selbstbestimmung" lautet, dann erläutert der Satz Der Mensch kann sich nur in der und durch die Geschichte verstehen nicht nur den verzeitlichten Horizont des Objektbereichs, sondern auch den reflexiven Status des zugrundegelegten Kulturbegriffs. ${ }^{17}$ Dennoch faßt diese Theorie das soziokulturelle Handeln nicht als dramatischen Prozeß, eine Betrachtungsart, die in der kulturanthropologischen und -soziologischen Modellbildung unseres Fin de siècle für Unruhe sorgt und neuerdings unter dem Kunstwort "Theatralität" die fragwürdige Statur einer kulturwissenschaftlichen Universalie annimmt. ${ }^{18}$ Für Dilthey ist dramatisch allein das "Leben", dessen

16 Diese Tendenz zeichnet sich in der von W. Frühwald, H. R. Jauß, R. Koselleck, J. Mittelstraß und B. Steinwachs verfaßten Denkschrift Geisteswissenschaften heute (Frankfurt/M.: Suhrkamp, 1991) $\mathrm{ab}$.

17 In Das Erlebnis und die Dichtung (213) heißt es unter Berufung auf Novalis, "daß die reale Psychologie oder Anthropologie den unendlichen Gehalt der menschlichen Natur nur an seiner Entwicklung in der Geschichte zu studieren vermag".

18 Vgl. Erving Goffman, The Presentation of Self in Everyday Life (New York: Doubleday, 1959); Victor Turner, The Anthropology of Performance, 2nd ed (New York: PAJ, 1992); Wolfgang Lipp, Drama Kultur. Teil 1: Abhandlungen zur Kulturtheorie. Teil 2: Urkul- 
Objektivationen jedoch in der kristallinen Form von Werken wahrgenommen werden, deren Abfolge auf der retrospektiv konstruierten historischen Achse unter dem Bild eines "Stufenganges" erscheint. Natürlich: Der Fluchtpunkt des verstehend erschlossenen historischen Raumes liegt in der Gegenwart. Diese soll aber - das ist Diltheys geschichtstheoretische Unterstellung - mit den früheren "Stufen" durch ein Überlieferungskontinuum in Verbindung stehen. Dieses wird von ihm nicht als träge dahinfließender Strom vorgestellt, sondern entspricht eher dem Bild einer aufhaltsamen, dennoch kumulativen Vorwärtsbewegung. Denn auch Dilthey geht von jenem Big Ditch in der Geschichte des europäischen Denkens aus, den die Rationalitätsschübe der neuzeitlichen Wissenschaften seit dem 17 . Jahrhundert zwischen modernem Weltbild und Metaphysik aufgerissen haben. Was in diesem Bruch endgültig untergeht, das ist die Metaphysik, während als Folge Dichtung und Wissenschaft in einen Gegensatz geraten, der - hier überläßt sich Dilthey einem geschichtsphilosophischen Traum des frühen Idealismus - irgendwann einmal überwunden werden wird:

Die poetische Phantasie wird lange Zeit unter die Herrschaft des Denkens geraten, sie wird oft in der Wissenschaft ihren Feind sehen, und erst wenn das Wissen an Leben und Geschichte heranrückt und die Dichtung an das Erfassen der ganzen Wirklichkeit, werden die Lebenserfahrungen des Dichters und das begriffliche Denken sich einander nähern. ${ }^{19}$

Das klingt wie eine vorweggenommene Antwort auf die Mitte unseres Jahrhunderts im britischen Universitätsmilieu vom Zaun gebrochene Zwei-Kulturen-Debatte. Die "ganze Wirklichkeit" läßt sich aber nach den knapp 40 Jahren, die uns heute von dieser Debatte trennen, selbst dann nicht mehr als haltbares Konzept verteidigen, wenn Literatur und Wissenschaft - wie es Aldous Huxley vorschwebte - sich wie kommunizierende Röh-

turen: Institutionen heute: Kulturpolitik (Berlin: Duncker \& Humblot, 1994). Die Deutsche Forschungsgemeinschaft hat 1995 einen neuen kulturwissenschaftlich-interdisziplinären Forschungsschwerpunkt vorgestellt und in einem intern verschickten Papier mit dem Titel "Theatralität: Theater als kulturelles Modell in den Kulturwissenschaften" erläutert.

19 Dilthey, Das Erlebnis 12. 
ren zueinander verhalten. Übrigens schließt das eine Annäherung à la Dilthey nicht aus, was der Tatsache zu entnehmen ist, daß der fundamentale Zweifel an der rationalen Erkennbarkeit der Wirklichkeit den philosophischen Diskurs (namentlich des Dekonstruktivismus) für poetische Tropismen wieder empfänglich gemacht hat.

Drittens: Wenn es überhaupt erlaubt ist, in Diltheys Theorie zwischen synchronen und diachronen Ebenen zu unterscheiden, so am ehesten dort, wo der Autor mit der Metapher des "Gewebes" (auch "Gespinst" oder "Netz") operiert. ${ }^{20}$ Schon im frühen Novalis-Essay heißt es, die verschiedenen philosophischen Disziplinen (Ethik, Ästhetik, Religionsphilosophie usw.) betrachteten "von verschiedenen Seiten dasselbe grenzenlose Gewebe von Erscheinungen", um "die inneren Zusammenhänge selber zu überblicken." ${ }^{21}$ Die Metaphorik des Webens und Knüpfens wird in den späteren wissenschaftsphilosophischen Hauptwerken beibehalten und gedehnt: so daß dieses Bildfeld bald die "Verwebung" zwischen Gesellschaft und Leben, bald die Synthesisleistung der einzelwissenschaftlichen Betrachtung bezeichnet, dann wieder das Zusammenführen der in dieser oder jener Disziplin zustandegekommenen Einsichten in einen allgemeinen Sinn- und Bedeutungszusammenhang. ${ }^{22}$

Der Gebrauch der Metapher ist vielseitig und weist dennoch deutlich in die Richtung einer als Text aufzufassenden und insofern lesbaren Struktur. Dem entspricht wohl auch die Operation des Verstehens, die - wie Dilthey selber sagt - "von außen nach innen" geht, von der gleichsam buchstabierten "Lebensäußerung" auf die "Erfassung des Innern, aus der sie hervorgeht" (VII, 82). Eine Figur des Nachschaffens, die auf der technischen Analyseebene jener Inversion der Rhetorik in Hermeneutik entspricht, von der schon Schleiermacher gesprochen hatte.

Das Bild des Gewebes weist - wie auch der äquivalente Textbegriff - auf ein In- bzw. Durcheinander heterogener Erscheinungen, dem insgeheim ein entzifferbares Muster zugrunde-

\footnotetext{
20 In bewußt metaphorischer Funktion: Dilthey, Das Erlebnis 8.

21 Dilthey, Das Erlebnis 214.

22 Vgl. etwa I, 422; VII, 102, 143 u. ö.
} 
liegt, das zu 'lesen' der philologisch geschulte Interpret besonders geeignet ist. Gewebe und Text sind räumliche Metaphern, die den Blick vom Prozeß ab- und auf synchrone Schnitte hinlenken. Der ethnologische Kulturanalytiker, der die Lebensformen einer fremden Welt wie einen Text zu entziffern sucht, verhält sich kaum anders als der wissenschaftliche Weber, dem Dilthey zur Aufgabe machte, die scheinbar beziehungslosen Zusammenhänge, in denen die Kultursubjekte blindlings handeln, als ein sinnvolles Ganzes darzustellen. Wie der Ethnologe, so begibt sich auch Diltheys Kulturinterpret auf eine von Außen nach Innen führende Bahn. Diese Richtung beruht in Diltheys Fall aber auf einer theoretischen Vorentscheidung, die das Zusammenknüpfen verschiedener einzelwissenschaftlicher Perspektiven zu einem - wie wir heute sagen würden - interdisziplinären Netz eher hemmt. Denn Dilthey hält an einer Psychologie des Sicheinfühlens fest, die vom Interpreten verlangt, den objektiven 'Gewebezusammenhang' als inneren Lebenszusammenhang verstehend nachzuerleben. Was selber der Erklärung bedarf, das Nacherleben, soll erklären, wie der Interpret dazu kommt, von der Gestalt der privilegierten Werke auf den "Geist" der Kultur zu schließen. Natürlich liegt dieser Auffassung ein symbolisches Darstellungsverhältnis zugrunde: aliquid stat pro aliquo; in der besonderen Einheit der Werkgestalt soll sich dem lesekundigen Blick die Signatur eines Zeitalters, einer kulturellen Einheit "offenbaren". Alte Diltheyaner haben die Geisteswissenschaften daher als eine Kunst der Ausdruckswahrnehmung, als "erweiterte Physiognomik" mißverstanden und das literarische Kunstwerk quasi zum Hyperzeichen und Ausdrucksphänomen einer Kultur, eines Zeitalters, einer Stilepoche erklärt. ${ }^{23}$ Eine Projektion des Besonderen ins Allgemei-

${ }^{23}$ Vgl. z. B. den programmatischen Aufsatz "Wesen und Bedeutung der Geistesgeschichte" von Walter Strich in dem von ihm herausgegebenen Jahrbuch für Geisteswissenschaften Die Dioskuren (München: Meyer \& Jessen, 1922) $7 \mathrm{f}$.: "Sprache ist Gestaltung, und die Art dieser Gestaltung enthüllt neben dem gesagten Inhalt, der hier ganz so wenig wie in der Kunst von der Form zu trennen ist, ein ganzes Weltbild, eine (...) Auffassung von dem Wesentlichen der Welt und des Menschen. Aufgabe der Geistesgeschichte ist es, zu zeigen, wie diese letzte Auffassung sich in dem unmittelbar Gegebenen offenbart." Zum poetischen Kunstwerk als “Ausdruck” der Kul- 
ne, die mit Diltheys Überlegungen nicht kompatibel ist, weil sie das komplexe Darstellungsverhältnis zwischen Text und Kontext, zwischen Werk und Kultur reduziert, um sich nicht lange bei den Zwischengliedern einer individuellen Lebensgeschichte (des Autors oder Künstlers), der Produktions- sowie Rezeptionsniveaus aufhalten zu müssen. Als Physiognomiker sieht der Interpret nicht blo $ß$ das gemachte Ding, wie es ist, oder den Verwertungsrahmen, in dem es z. B. als Funktion einer Handlung aufgeht - und sei es in der von I. A. Richards und K. Burke geprägten symbolischen Fassung des dancing of an attitude. Sein ästhetischer Blick sieht das Ding vielmehr als Bild, dessen symbolische Struktur den gewöhnlichen philologisch-historischen Erklärungen, die in den Bahnen der Zweck-Mittel-Rationalität verharren, inkommensurabel ist. So wichtig dieser theoretische, will sagen anschauungssüchtige Überschwang als Basis für die Kritik am wissenschaftlichen Rationalitätskult auch war, der notwendigen Verknüpfung zwischen der empirischen historisch-philologischen - Sachforschung und der symbolischen - reflexiv-hermeneutischen - Kontextualisierung stand er im Wege. Denn die Ausdruckswahrnehmung sucht, ausgehend vom Dogma des "reinen Sehens" - eine Unmittelbarkeit herzustellen, die Diltheys Doktrin vom Lehren und Lernen des Sehens unterbietet.

Die drei hier in grober Manier skizzierten Problemfelder hat die Dilthey-Zeit den Kunstwissenschaften, denen ich die von den Philologien sich lösenden Literaturwissenschaften zurechne, als zweideutiges Familienerbe hinterlassen. Die These vom großen Zusammenhang der Lebenswelt, hinter der vielleicht noch ein Schatten jener Kosmosmetapher zu ahnen ist, die sich als 'Buch der Welt' in die Dokumente früherer Gelehrsamkeit eingeschrieben hat, stand einem interdisziplinären Organisationskonzept nicht völlig fremd gegenüber. Es sind ja, um mit Dilthey zu reden, verschiedene Seiten ein und desselben "Gewebes", auf deren Entzifferung sich die Neugier der Einzelwissenschaften richten sollte, ohne in jenes partikulare Nebeneinander zu zerfallen, das mit unfreiwilliger Komik Hermann Use-

tur vgl. Oskar Walzel, Gehalt und Gestalt im Kunstwerk des Dichters (Berlin: Akad. Verl.-Ges. Athen, 1923) 132 u. 142. 
ners Satz illustriert: "Selbst ist der Mann gilt vom Philologen mehr noch als von anderen." ${ }^{24}$ Die Theorie der Geisteswissenschaften enthält zwar keinen Hinweis auf eine interdisziplinäre Organisation kulturwissenschaftlicher Einzelforschungen, läßt sich aber durchaus als historische Variante einer entsprechenden Metatheorie verstehen. ${ }^{25}$

Wilhelm Scherers polemischer Hinweis auf den ökonomischen Tauschwert des Gedichts sollte wohl vor allem daran erinnern, daß Kultur bzw. Literatur nicht nur eine ideelle, sondern auch eine materielle Dimension besitzt. Das war natürlich auch Dilthey nicht unbekannt, der von Anfang an darauf bestand, daß sich die Erkenntnisweisen der Geisteswissenschaften nicht nur auf Texte beschränken dürfen, wenn sie die ganze rätselhafte Gestalt des "Lebens" in der Anschauung der überkommenen Kulturobjektivationen nacherleben wollten. Wie sehr auch immer der Lebensbegriff diese Theorie trübt, er läßt doch die Ahnung zu, daß im Grunde der idealistische "Geist" als Ausgangspunkt für Diltheys Wissenschaftsgrundlegung denkbar ungeeignet war. Denn "Leben" signalisiert ein dynamisches Integral der Kultur, das sich nicht wie der "Geist" dem Begriff erschließt, sondern der (ästhetischen) Anschauung bedarf, um in seiner Fülle, d. h. nicht nur durch die "Nebel" der Abstraktion, wahrgenommen zu werden. Natürlich ist das problematisch für eine Betrachtungsart, die wie die wissenschaftlichanalytische auf scharfe Distinktionen und Begriffe angewiesen ist. Diltheys Annahme, die Weltanschauungstypen gingen unmittelbar aus dem "Leben" hervor, haben ihm den Weg zur methodologischen Vermittlung zwischen empirisch-analytischen und hermeneutischen Verfahren verstellt.

${ }^{24}$ Hermann Usener in seiner Rektoratsrede "Philologie und Geschichtswissenschaft" von 1882, Vorträge und Aufsätze (Leipzig: Teubner, 1907) 22.

25 Vgl. zu diesem Komplex Stefan Haas, Historische Kulturforschung in Deutschland 1880-1930: Geschichtswissenschaft zwischen Synthese und Pluralität (Köln: Böhlau, 1994). 
Allgemeine Wissenschaftsgrundlegungen sind, wie man gerechterweise sagen muß, nicht allein vom häuslichen Standpunkt der Fachmethodik aus zu beurteilen. Warum dann überhaupt Dilthey, wenn es doch um den Status der Literaturwissenschaften geht? Als Dilthey an der Einleitung in die Geisteswissenschaften schrieb, war die Literaturbetrachtung noch eingebunden in den großen Haushalt der Nationalphilologien, der sich nicht allein auf die Rekonstruktion älterer Sprach- und Literaturstufen beschränkte. Hermann Paul, der 1891 den ersten Band des Grundrisses der Germanischen Philologie herausgab, hatte zum Ziel, eine "allgemeine Kulturwissenschaft" vorzustellen, die wie eine Herrschaftsvilla mit eng angeschlossenen $\mathrm{Ne}$ bengebäuden angelegt ist: auf der einen Seite Sprachen und Literaturen, auf der anderen Künste, Wirtschaft, Rechtssystem, Religion etc. - in graphischer Vereinfachung:

NATIONALPHILOLOGIE ALS ALLGEMEINE KULTURWISSENSCHAFT

$\begin{array}{lll}\text { Sprache: } & \text { Literatur: } & \text { [Kontext]: } \\ \text { Schriftkunde } & \text { Heldensage } & \text { Künste } \\ \text { Geschichte der } & \text { Geschichte der } & \text { Sitte \& Recht } \\ \text { Einzelsprachen } & \text { Einzelliteraturen } & \text { Mythos \& Kultur } \\ & & \text { Kriegswesen } \\ & & \text { Ökonomie }\end{array}$

Wie diese Einzelkomplexe in einen wissenschaftlich gegliederten Zusammenhang zu bringen sind, sucht Paul in der "Methodenlehre" zu erklären, die manche Einsicht August Boeckhs Enzyklopädie und Methodologie der philologischen Wissenschaften verdankt. Paul spricht wie Dilthey von der wissenschaftlichen Arbeit als "Konstruktion", die das lückenhaft Vorgefundene ergänzen muß, um - wie der Philolog Boeckh formuliert hatte - "was nicht als Ganzes erscheint, zu einem Ganzen (zu) vereinigen". ${ }^{26}$ Die historische Erkenntnis fußt deshalb

26 Hermann Paul, "Methodenlehre", Grundriss der germanischen Philologie, Bd. I/2 (Straßburg: Trübner, 1901) 163; August Boeckh, Enzyklopädie und Methodologie der philologischen Wissenschaften, ed. Ernst Bratuscheck (Leipzig: Trübner, 1877) 15. Zur synthetisieren- 
nicht auf einem in sich selbst ruhenden erfahrungswissenschaftlichen Prinzip, sondern bedarf einer theoretischen Grundlegung, die der Sprachhistoriker Paul - mit der Zeit gehend - in der Psychologie gesucht hat. ${ }^{27}$

Pauls monumentaler Grundriss läßt sich als Antwort auf die Krise des Historismus verstehen, die von jener Sezessionsbewegung innerhalb der Philologien beschleunigt wurde, die in die kleineren Haushalte autonomer Literaturwissenschaften münden sollte. Die Bezeichnung "Literaturwissenschaft", die schon in den 20er Jahren des 19. Jahrhunderts auftauchte, hatte ihre eigentliche Bestimmung noch nicht gefunden, und es scheint, daß sie seitdem sich durch das definiert, was sie noch nicht ist. Eine Charakterisierung, die gut zu jener romantisch-idealistischen Idee einer progressiven Universalpoesie passen mag, die dem kleinen Haushalt das hochmoderne Aussehen einer emsig offen gehaltenen Baustelle verschafft. Zur Zeit von Pauls Grundriss aber hatte die "Literaturwissenschaft" noch den Fanfarenklang einer Bewegung, die sich aus der mütterlichen Umarmung der klassischen Philologie losreißen wollte, ohne freilich ihrer vielgelobten wissenschaftlichen Strenge völlig zu entraten, die sich in Historischer Grammatik, Lexikographie, Textkritik und -exegese erschöpfte.

Die Sezessionisten konnten noble Gründe für ihren Selbständigkeitsdrang ins Feld führen, Gründe, die ich hier kurzerhand unter der kulturellen Idee der ästhetischen Autonomie zusammenfassen möchte. Diese Idee rechtfertigte die Spezialisierung literaturwissenschaftlicher Auslegung auf ästhetische Texte und verwies die historisch-philologische, z. B. die textgenetische, historisch-funktionalistische Erklärung des poetischen Einzelwerks in die propädeutischen Randgebiete. Analyse und Deutung des Einzelwerks ohne Preisgabe des philologischen Handwerks - das war der Kern dieser Literatur-Wissenschaft, um diese von der konventionellen Literatur-Geschichte zu un-

den Funktion der wissenschaftlichen Darstellung vgl. Jürgen Fohrmann, "Literaturgeschichtsschreibung als Darstellung von Zusammenhang", Von der gelehrten zur disziplinären Gemeinschaft, ed. J. Fohrmann und W. Voßkamp (Stuttgart: Metzler, 1987) $174 \mathrm{ff}$.

27 Zur Kritik vgl. Karl Vossler, Geist und Kultur in der Sprache (Heidelberg: Winter, 1925) $5 \mathrm{f}$. 
terscheiden. Dennoch, was unterschieden wird, muß, soll ein Ganzes daraus werden, auch wieder zusammengefügt werden. Und das war die Aufgabe der Methodologie, die zunächst (Werk-)Analyse und (Geschichts-)Synthese schied, um eine Verfahrensrangfolge des einen gegenüber dem andern und schließlich ihrer Komplementaritätsbeziehungen zu rechtfertigen. Stark abstrahiert und graphisch verkürzt erscheint unter dieser Voraussetzung das Schema des kleinen Haushalts in folgendem Bild: ${ }^{28}$

\begin{tabular}{|l|l|l|}
\hline \multicolumn{3}{|c|}{ Literaturwissenschaft } \\
\hline Aufgabe: & Methoden: & Begriffsbildung: \\
\hline Analyse & Werk-Interpretation & \multirow{2}{*}{ Kritisches } \\
\hline Synthese & Vgl. Literarhistorie & Werturteil \\
\hline
\end{tabular}

Es scheint, als habe der kleine Haushalt Kultur und Sprache vor die Tür gesetzt. Doch in Wahrheit geht es um eine Akzentverschiebung zugunsten einer neuen, Autonomie beanspruchenden Disziplin; mit den Worten des Novalis- und HamannExperten Rudolf Unger aus einem Aufsatz von 1914 um den:

systematischen Auf- und Ausbau der neueren deutschen Literaturwissenschaft, über ihr Verhältnis zur älteren deutschen [Germanische Philologie], zur antiken [Klassische Philologie] und zur sogenannten vergleichenden oder internationalen Literaturgeschichte, zur Kunstwissenschaft, zur Sprachwissenschaft, zur Philosophie, zur Kulturgeschichte und modernen Soziologie. ${ }^{29}$

28 Ich nehme in die Graphik Unterscheidungen und Begriffe auf, die Konzepte aus Ernst Elsters Prinzipien der Literaturwissenschaft (Halle: Niemeyer, 1897) variieren.

29 Rudolf Unger, Aufsätze zur Prinzipienlehre der Literaturgeschichte (Berlin: Junker \& Dünnhaupt, 1929) 48. Vgl. zu den Konsolidierungsschwierigkeiten des neuen Fachs den Überblick von Holger Dainat, "Von der Neueren deutschen Literaturgeschichte zur Literaturwissenschaft: Die Fachentwicklung von 1890 bis 1913/14", Wissenschaftsgeschichte der Germanistik im 19. Jahrhundert, ed. J. Fohrmann und W. Voßkamp (Stuttgart: Metzler, 1994) 494-537. 
Ungers Programm wendet sich bewußt gegen die positivistische Wissenschaftstradition des 19. Jahrhunderts. ${ }^{30}$ Seine Worte machen deutlich, daß sich das Neue noch nicht konsolidiert hat, und deuten zugleich jene ungeklärten Beziehungen zwischen Kernfach und sogenannten Hilfswissenschaften an, die sich an den immerzu wechselnden Konfigurationen der Geschichte der Literaturwissenschaften bis heute ablesen lassen. Ungers Ziel war es, den Entwurf einer selbständigen Literaturdisziplin in den großartigen Rahmen einer umfassenden "Kulturwissenschaft" einzufügen, die den allgemeinen "Kulturprozeß" von innen her - auf dem Weg "teleologischen Begreifens" zur Erkenntnis zu bringen hatte. Literaturwissenschaft, Sozialpsychologie, Philosophie und Ethnologie (Völkerkunde) sollten in diesem Programm zu einer Einheit verschmelzen, der ein ebenso einheitlicher Kulturbegriff entsprach. Der Begriff der Einheit schließt den der prozessualen Entfaltung freilich nicht aus. Diesen Proze $\beta$ zu ordnen, "dem uferlosen Ozean der Geschichtlichkeit einen Sinn abzuringen", ist Aufgabe der Kulturwissenschaft und der in diese eingeschlossenen Literaturforschung. Doch tritt das Interesse an der Interaktion zwischen Kultur- und Literatursystem, auf die Diltheys Gewebe-Metapher verwies, hinter das an der Werkeinheit zurück: Einfühlen, Verstehen, Nachschaffen lauten auch hier die favorisierten Annäherungsweisen. ${ }^{31}$ Auf den positivistischen Empirismus antwortet Unger mit der Forderung, Einfühlungshermeneutik (Dilthey) und kunstwissenschaftliche Stiltypologie (Wölfflin) zu fusionieren; eine Forderung, an die bald darauf Oskar Walzel mit seinem einflußreichen Buch Gehalt und Gestalt im Kunstwerk des Dichters (1923) direkt und ausführlich angeknüpft hat. ${ }^{32}$

Wir kennen das Problem der Einfühlungshermeneutik. Sie hat einseitig das Prinzip der Kongenialität gegen jene technische Seite der Hermeneutik gewendet, die den lehr- und lern-

30 Vgl. auch seinen Aufsatz "Moderne Strömungen in der deutschen Literaturwissenschaft", Die Literatur 26 (1923/24): $65 \mathrm{ff}$.

31 Unger 29 ff. Eckpfeiler der "Kulturwissenschaft", die Unger nicht scharf von "Geisteswissenschaft" unterscheidet, sind für ihn Kulturphilosophie (F. Nietzsche, G. Simmel), Ethnologie (A. Bastian), Sozialpsychologie (A. Vierkandt) und Kulturgeschichte; 24.

32 Oskar Walzel (vgl. Anm. 23) 15 u. ö. 
baren Methoden der Textanalyse gewidmet ist. ${ }^{33}$ Auch Unger war ein Parteigänger dieser halbierten Verstehenslehre. "Gleiches wird durch Gleiches erkannt", resümiert er und fügt hinzu, daß wir "in der Geschichte nur unser eigenes Leben, unser eigenes Wesen wiederfinden." ${ }^{34}$ Es geht ihm ums Eigene, nicht ums Befremdliche oder gar um Verfremdung. Das Eigene, nämlich die kanonische Nationalliteratur im Prozeß der Aneignung aktiv weiter zu entwickeln, ist der eigentliche Zweck dieser Spielart kultur- bzw. literaturwissenschaftlicher Sinnfindung.

Das hier skizzierte Konzept der Kulturwissenschaft war noch in höchstem Maß jenem normativen Singular "Kultur" verpflichtet, den Fritz Mauthner in seinem 1910/11 erschienenen sprachkritischen Wörterbuch der Philosophie mit einem "Sollzustand" identifiziert und scharf vom deskriptiven Plural "Kulturen" unterschieden hat. ${ }^{35}$ Auch Unger arbeitet mit zwei Begriffen, nämlich mit der Dichotomie zwischen ideeller und materieller Kultur. Die Kultursysteme und -prozesse, die er in seinen prinzipienwissenschaftlichen Beiträgen Mitte der 20er Jahre der geistesgeschichtlichen Betrachtungsart zuschlägt, sind Teil dessen, was er den "ideellen Oberbau" nennt. Und dieser ist vom "staatlich-sozialökonomischen Unterbau", mit dem er in einer nur vage bezeichneten Wechselbeziehung stehen soll, zu unterscheiden. Der "ideelle Oberbau", zu dem die poetische Literatur gehört, soll seine eigenen "geistigen", ins Überhistorische (ins Kosmisch-Menschheitliche) verweisenden immanenten Gesetze besitzen. Ihm wird daher nur eine "theoretisch-kontemplative Geisteshaltung" gerecht, die Unger mit kulturchauvinistischem Gestus dem "westlichen Positivismus (und damit)

33 Vgl. die Darstellung der ungeteilten Hermeneutik und ihre Anschließbarkeit an strukturalistische Verfahren in Manfred Franks Artikel “Textauslegung”, Erkenntnis der Literatur: Theorien, Konzepte, Methoden der Literatumissenschaft, ed. D. Harth und P. Gebhardt (Stuttgart: Metzler, 1982) $123 \mathrm{ff}$.

34 Unger 30.

35 "Kultur ist der Sollzustand, zu welchem sich ein Mensch oder ein Volk hinaufentwickeln mag; die Kulturen der verschiedenen Völker bezeichnen einen Istzustand." Fritz Mauthner, Wörterbuch der Philosophie: Neue Beiträge zu einer Kritik der Sprache, Bd. 2 (Zürich: Diogenes, 1980) 42. 
verbündeten modernen Intellektualismus" gegenüberstellt. ${ }^{36}$ Zwar sucht dieses geistesgeschichtliche Konzept mit ideologischem Starrsinn den längst dahingeschwundenen Kultwert der Dichtung allen Auflösungserscheinungen zum Trotz festzuhalten. Dennoch verfehlt es die von Unger erträumte "unteilbare Literaturwissenschaft", da er nicht erklären kann, wie der von ihm entfaltete Fächer "historisch-philologischer", "kulturgeschichtlich-soziologischer", "literatur-ethnologischer", "kunstwissenschaftlich-ästhetischer" und "stiltypologischer" Betrachtungsarten in einer konsistenten Fachmethodik unterzubringen ist. ${ }^{37}$

Das Bild der kulturwissenschaftlich-literaturwissenschaftlichen Diskussion zur Zeit Ungers bliebe ein Zerrbild, würde nicht jener gleichzeitigen Neuansätze gedacht, die in der gegenwärtigen Suche der Geisteswissenschaften nach einem neuen Selbstbewußtsein als Schlüsseldiskurse anzusehen sind: Semiotica universalis und Historische Anthropologie. Beide Diskurse waren von Anfang an interdisziplinär ausgerichtet und haben unabhängig voneinander die symbolischen, in sprachlichen und bildlichen Zeichen manifesten Strukturen und Funktionen kultureller Artefakte ins Zentrum der Analyse gerückt. Ich erwähne hier nur die Arbeiten von Roman Jakobson und der Warburg-Schule, die die Grundlagen für eine Kulturwissenschaft geschaffen haben, die sich als offenes System versteht. ${ }^{38}$ Warburg hat die Theorie "reinen Sehens", die Wölfflin entwickelte, als unzulässige Abstraktion zurückgewiesen. Ihm ging es nicht um die geistige Repräsentationsfunktion des künstlerischen

36 Unger $216 \mathrm{ff}$.

37 Unger 224.

38 Zur Bedeutung dieser Position in der Grundlagendebatte vgl. die Einleitung von Elmar Holenstein zu dem von ihm herausgegebenen Sammelband: Roman Jakobson, Semiotik: Ausgewählte Texte 1919-1982 (Frankfurt/M.: Suhrkamp, 1988), sowie folgende Aufsätze: Peter Burke, "Aby Warburg as Historical Anthropologist", Aby Warburg: Akten des internationalen Symposiums Hamburg 1990, ed. H. Bredekamp, M. Diers und C. Scholl-Glass (Weinheim: VCH, 1991) 39 ff.; Sigrid Weigel, “Aby Warburgs 'Schlangenritual': Korrespondenzen zwischen der Lektüre kultureller und geschriebener Texte", Paragrana 3 (1994): 9 ff. Alle genannten Publikationen enthalten reichhaltige bibliographische Hinweise. 
Einzelwerks, sondern darum, die systematische Analyse der ästhetischen Ausdruckswerte in jener anthropologisch-historischen Dimension zu entfalten, die von der mythischen bis zur rationalen Weltorientierung führt: "vom Fetisch bis zum Drama". ${ }^{39}$ Mit den Begriffen des "Symbols" und der "symbolischen Form", deren philosophische Grundlegung dem mit der Warburg-Schule eng verbundenen Philosophen Ernst Cassirer zu verdanken ist, haben Warburg und seine Schüler ein Konzept gefunden, das geeignet schien, das Diltheysche Geist-LebenDilemma zu überwinden. Denn der Symbolbegriff verweist auf einen begrifflich zu fassenden und einen ästhetisch erfahrbaren Pol im Artefakt, deren Zusammenspiel ein energetisches, über das Werk hinaus wirkendes Spannungsfeld erzeugt. In der Erläuterung Edgar Winds:

Die kritische Phase liegt (...) in der Mitte, dort, wo das Symbol als Zeichen verstanden wird und dennoch als Bild lebendig bleibt, wo die seelische Erregung, zwischen diesen beiden Polen in Spannung gehalten, weder durch die bindende Kraft der Metapher so sehr konzentriert wird, daß sie sich in Handlung entlädt, noch durch die zerlegende Ordnung des Gedankens so sehr gelöst wird, daß sie sich in Begriffe verflüchtigt. ${ }^{40}$

Den Symbolbegriff bezeichnet demnach eine Doppelcodierung, die sich auf wissenschaftlicher Ebene einer zweifachen Operation erschließt: der semantischen, auf die Zeichenstruktur bezogenen Analyse und der ästhetischen Analyse, die dem energetisch wirkenden (lebendigen) Bild gewidmet ist. Die Symbolwahrnehmung trennt nicht zwischen Begriff und Anschauung. Sie gibt dem begrifflichen Zeichen-Verstehen kein höheres Recht als der ausdrucksbezogenen Bild-Anschauung, sondern führt beide Pole unter dem Symbolbegriff zusammen. Symbole sind nach dieser Theorie nicht an und für sich und daher weder durch Einfühlung (Dilthey) noch durch "reines Sehen" (Wölfflin) der Gestaltsqualität zu erschließen. ${ }^{41}$ Bildsymbole sind nicht

39 Aby Warburg, "(Rede vor dem Kuratorium der Kulturwissenschaftlichen Bibliothek, 21.08.1929)”, Aby M. Warburg: Ausgewählte Schriften und Würdigungen, ed. D. Wuttke (Baden-Baden: Koerner, 1980) 307.

40 Edgar Wind, "Warburgs Begriff der Kulturwissenschaft und seine Bedeutung für die Ästhetik”, 1931, Wuttke 410.

41 Wind 168: "Der Forscher (...) kann sich nicht dem Glauben hinge- 
nur Geformtes, sie sind vielmehr ihrerseits wirksame (energetische) Agenzien der Formgebung, deren wissenschaftlich reflektierbare Rekonstruktion Warburg mit dem anthropologischen Leitgedanken eines wandelbaren, epochenübergreifenden "Bildgedächtnisses" (Mnemosyne) in Beziehung gesetzt hat. Dieses Projekt basiert auf einer interdisziplinären Kommunikation, die sich - wie es bei E. Wind heißt - der "unauflöslichen Verflochtenheit" des Einzelwerks mit der "Gesamtkultur" versichern sollte und zu diesem Zweck energisch in die Gebiete der Kulturgeschichte, der Religionswissenschaft, der Philosophie und der Philologien auszugreifen hat. ${ }^{42}$

Es ist hier nicht der Ort, diese Theorie und ihre Variationen bei Autoren wie Gombrich, Panofsky und Wind - en détail darzustellen oder gar zu kritisieren. ${ }^{43}$ Hier konnten nur einige wenige Aspekte zur Sprache kommen, die andeuten sollten, daß sich bereits in den 20er und 30er Jahren jener Umbau der Kulturwissenschaften abzeichnete, der heute unter den Leitbegriffen der Symbolinterpretation und des ethnologischen Blicks eine Renaissance erlebt. Bemerkenswert ist: Den skizzierten Neuansatz haben Erfahrungen angeregt, die auf die Begegnung mit einer außereuropäischen Kultur, der Kultur der PuebloIndianer Neu-Mexicos, zurückzuführen sind. "Denn Warburg verdankte es Amerika, daß er lernte, die europäische Geschichte mit den Augen eines Anthropologen zu sehen." ${ }_{44}$

ben, daß seine Betrachtung eines Bildes ein einfaches Anschauen, ein unmittelbares Sicheinfühlen sei."

42 Wind 168.

43 Weiterführende Untersuchungen: Pierre Bourdieu, Zur Soziologie der symbolischen Formen (Frankfurt/M.: Suhrkamp, 1983); Bernhard Buschendorf, "Enthusiasmus und Erinnerung in der Kunsttheorie Edgar Winds", Mnemosyne: Formen und Funktionen der kulturellen Erinnerung, ed. A. Assmann und D. Harth (Frankfurt/ M.: Fischer, 1991) $319 \mathrm{ff}$. Zur Kritik an Warburg vgl. die Aufsätze von P. Burke und S. Weigel (Anm. 38).

44 Fritz Saxl, "Warburgs Besuch in Neu-Mexico", Wuttke (Anm. 39) $317 \mathrm{ff}$. 
Seit 1933 ging die Literaturwissenschaft in Deutschland ihre eigenen Wege. Die NS-Germanistik erniedrigte sie zum "Dienst am inneren Reich der Deutschen" und hat sich um die von Unger und anderen hinterlassenen ungelösten theoretischen Probleme den Teufel geschert. ${ }^{45}$ Ich übergehe hier die Frage, in welchem Maß diese Erniedrigung Folge einer schon länger angebahnten Selbsterniedrigung war, zumal sich die jüngste Fachhistorie dazu bereits ausführlich zu Wort gemeldet hat. ${ }^{46}$ Eines darf indessen nicht übersehen werden: Die kulturchauvinistischen Äußerungen Ungers, Walzels und anderer Vertreter der germanistischen Literaturwissenschaft in den 20er Jahren sind Belege für ein Konzept der "Kulturwissenschaft", das nicht nur innerhalb nationalkultureller Grenzen verharrte, sondern sich offenbar auch verpflichtet sah, immer wieder - mit den Mitteln gebetsmühlenhaft wiederholter Distinktionen vor allem gegenüber der Kultur des westlichen Nachbarn - den nationalen Standpunkt zu beteuern. 1942 schrieb der nun im Exil lebende Hausphilosoph der Warburg-Schule der Kulturwissenschaft ins Stammbuch: Die Kultur ist ein Tun, das seines Zieles "Verwirklichung der Freiheit" - niemals sicher ist. ${ }^{47}$

Doch zurück zu den systematischen Fragen. Die heute erhobene Forderung, die Literaturwissenschaften in ein kulturwissenschaftliches System einzubinden, kann - wie zu zeigen war bis auf die Anfänge dieser Fachdisziplin zurückgeführt werden. Damit soll nicht einer Anknüpfung an Früheres das Wort geredet werden. Der Rückblick ist Erinnerung und möchte auf eine

45 Gerhard Fricke hat im Vorwort des 1944 von ihm herausgegebenen 3. Bandes der Gesammelten Studien Ungers das Wort vom "Dienst am inneren Reich" geprägt; ich zitiere nach dem 1966 (Darmstadt: Wiss. Buchges.) erschienenen Nachdruck, 8.

46 Vgl. u. a. Hartmut Gaul-Ferenschild, National-völkisch-konservative Germanistik: Kritische Wissenschaftsgeschichte in personengeschichtlicher Darstellung (Bonn: Bouvier, 1993).

47 Zum Kulturchauvinismus gehören nicht zuletzt Walzels wiederholte Abgrenzungen gegenüber der "lateinischen" Kultur; vgl. Walzel (Anm. 23) 377 u. ö. Das letzte Zitat nach Ernst Cassirer, Zur Logik der Kulturwissenschaften, 1942 (Darmstadt: Wiss. Buchges., 1971) $104 \mathrm{ff}$. 
Problematik aufmerksam machen, die sich damals wie heute nicht mit einem Streich lösen läßt. "Die moderne Literaturwissenschaft - d. h. die der letzten fünfzig Jahre - ist ein Phantom." Gegen dieses 1948 von Ernst Robert Curtius formulierte Urteil läßt sich Nennenswertes nicht einwenden. ${ }^{48}$ Die Nationalphilologien haben dieses Verdikt überlebt - gewiß nicht zu ihrem Vorteil.

Die inzwischen unter dem alten Fahnenwort "Kulturwissenschaft" daherkommende Forderung nach inter-, wenn nicht gar transkultureller Erweiterung, die sogar noch Curtius' Traum einer Europäischen Literaturwissenschaft überbietet, steigert in nicht unerheblicher Weise den Versuchscharakter literaturwissenschaftlicher Forschungen. Was "Kultur", was "Literatur" ist, hängt davon ab, wie die mit diesen Kollektiva bezeichneten Phänomene begrifflich gefaßt werden. Daß sie ineinander aufgehen sollen, bedarf allerdings genauer Prüfung. Denn vom literarischen Text aus gesehen kann die "Kultur", in deren Rahmen er entsteht, verwertet wird oder zirkuliert, nur als Kontext gelten. Was sich der Orientierungssuche anbietet, das ist daher der Abschied von einer allein seligmachenden Fundierung in irgendwelchen holistischen Konzepten und die Anerkennung von Teiltheorien mit beschränkter Reichweite, die ebenso vielen Aspekten jener Literaturen und Kulturkontexte entsprechen, die zur Wahl stehen bzw. zur Interpretation herausfordern.

Den Methodenpluralismus zu beklagen, lenkt nur von der Tatsache ab, daß es moralische oder politische Argumente sind, die die faktische Vielheit der Arbeitsweise auf eine bindende Idee - der Bildung, der Wertsteigerung, der Identitätsstiftung etc. - verpflichten wollen. Solchen Ideen liegen in der Regel Kultur- bzw. Literaturkonzepte zugrunde, die in teleologischen oder evolutionistischen Vorstellungen befangen bleiben oder zumindest von einer homogenen Struktur des jeweiligen Systems ausgehen. Wenn wir Kulturen und ihre literarischen Subsysteme, um sie beschreiben zu können, als "Tatsachen" betrachten, dann gilt auch in diesem speziellen Rahmen Nelson

48 Ernst R. Curtius, Europäische Literatur und lateinisches Mittelalter, 7. Aufl. (Bern: Francke, 1969) 22. 
Goodmans Bemerkung: "Sie sind ebenso theoriegeladen, wie wir von unseren Theorien hoffen, daß sie tatsachengeladen sind." ${ }^{49}$ Will sagen: Der Aspekt, unter dem der Wissenschaftler literarische Texte als Komponenten eines kulturellen Handlungskomplexes betrachtet, realisiert eine unter mehreren möglichen Anschauungsweisen (in der Bedeutung von theorein), die der Gegenstand ihm nahelegt. Es sind die unter dem Gesichtspunkt der Zweckmäßigkeit entstehenden, die pragmatischen Teiltheorien, die es ihm erleichtern, seine "Konstruktion", um einer nachvollziehbaren "Version" willen, von der universalistischen Fokussierung Diltheys auf die "ganze Wirklichkeit" zu lösen. Was zweckmäßig ist, liegt auf der Hand, wenn man die Kollektiva "Kultur" und "Literatur" nicht mit den Forschungsgegenständen verwechselt, sondern als das versteht, was sie sind: holistische Signifikanten, die heute jedoch - angesichts gesteigerter kultureller und literarischer Austauschprozesse - nicht mehr vom theoretischen Standpunkt homogener Werthierarchien aus zur Anwendung kommen sollten. Der Begriff der Version, von Goodman ins Zentrum seiner Theorie des Worldmaking gerückt, ist direkt an die Arbeit des Kultur- und Literaturforschers anschließbar, da er, wörtlich genommen, nichts anderes als die Übertragung in eine Lesart bezeichnet, und Lesarten teilen die Eigenschaften aller Kommunikationsprozesse: Sie heischen Zustimmung auf Widerruf.

Damit bin ich an einem Punkt angelangt, in dem kultur- und literaturwissenschaftliche Recherchen einander besonders nahe kommen. Denn Lesarten im wissenschaftlichen Sinn beruhen nach wie vor auf Methoden der Textexplikation, die von den hermeneutischen Kernfragen, wie der Gegenstand zu bestimmen und zu erkennen ist, nicht absehen können. Für den Literatur- oder Textwissenschaftler mag es daher eine besondere Genugtuung sein, daß sich die Kulturanthropologie - ich denke hier in erster Linie an die Spielart der "interpretive anthropology" - literaturkritischer Modelle und Methoden bedient. Die Strukturen selbst einer fremden Kultur erscheinen in diesem Erklärungsspiel der Analogien wie ein Text, der sich, so scheint

49 Nelson Goodman, Weisen der Welterzeugung (Frankfurt/M.: Suhrkamp, 1984) $120 f$. 
es, nach den modi operandi der klassischen Kritik rekonstruieren und entziffern läßt. Eine "neue Philologie" tritt auf den Plan und erinnert, auch ohne dies ausdrücklich zu erwähnen, an die Herkunft der Ethnologie aus der klassischen Philologie. ${ }^{50}$

In a multicultured world, a world of multiple epistemologies, there is need for a new philologist - a specialist in contextual relations - in all areas of knowledge in which text-building (...) is a central activity: literature, history, law, music, psychology, trade, even war and peace. ${ }^{51}$

Die Ethnographie hat damit bewußt den Schritt vom Grammatikmodell der gesprochenen Sprache, an dem sich noch Edmund Leachs Kulturanalyse orientierte, zur Texthermeneutik vollzogen. ${ }^{52}$ Was aber heißt hier "Text"? Der kulturanalytische Begriff, der nur oberflächlich Diltheys Gewebemetapher ähnelt, bezeichnet einen Handlungsablauf, den der wissenschaftliche Beobachter aufzeichnet, um das Aufgezeichnete in eine Lesart zu übertragen. Wie der philologische Leser Form und Gehalt des schriftlichen Textes aufeinander bezieht, um eine erste semantische Analyse $\mathrm{zu}$ wagen, und wie er die so gewonnenen Deutungshypothesen anhand von Kontextlektüren überprüft, so ähnlich verfährt der Kulturanalytiker. Der Unterschied ist: Sein Material ist fließend, und doch enthält es für die Akteure etwa innerhalb eines religiösen Rituals - einen über den Handlungsvollzug hinaus andauernden Sinn, den der Ethnograph mithilfe von Operationen herauszufinden sucht, die ähnlich wie die des Philologen vom Ereignis zu Kontexten und von dort wieder zurück zum Ereignis führen. Der Einstieg in die Analyse des beobachtbaren Handlungszusammenhangs kann an einem beliebigen Detail ansetzen, um von dort, nach Art eines kommunikativen Zirkels, in weitere Zusammenhänge vorzustoßen, bis das Ganze des Ereignisses begriffen ist. Auch für diese

50 Vgl. die Hinweise in meinem Essay "Der Forscher als Schamane", Ruperto Carola: Forschungsmagazin der Universität Heidelberg 3 (1995): $4 \mathrm{ff}$.

51 Alton Becker, zit. nach Geertz (Anm. 5) 32.

52 Vgl. E. Leach, Culture and Communication: The Logic by Which Symbols are Connected (Cambridge: Cambridge UP, 1976) 10: "I assume (...) it is just as meaningful to talk about the grammatical rules which govern the wearing of clothes as it is to talk about the grammatical rules which govern speech utterances." 
Umschreibung des kulturanalytischen Prozesses kann sich der Ethnograph auf die Philologie berufen: Geertz z. B. vergleicht sein Verfahren der "dichten Beschreibung" mit der kritischen Lektüre eines Gedichts und verweist auf Leo Spitzers Methode der explication de texte. ${ }^{53}$ - Ich möchte die Analogiesuche hier nicht weitertreiben. Sie wäre leicht zu befriedigen; und zwar weit über die Familienähnlichkeiten mit der Philologie hinaus, da die Kulturanthropologie einen in der Zunft heftig umstrittenen Zug ins Literarische besitzt und manche ihrer Fundamentalkonzepte - z. B. Arnold van Genneps rites de passage - verschwiegene Vorbilder in der klassischen Poetik haben. ${ }^{54}$ Ein ergiebiges Feld für interdisziplinäre Untersuchungen!

Meine letzte Überlegung gilt der Frage, woran die Literaturwissenschaft anknüpfen kann, um sich im Konzert einer interdisziplinären Kulturwissenschaft zu qualifizieren. Mehr als eine sehr knappe Glosse kann ich hier freilich nicht bieten und beginne zunächst mit einem Überblick, der schematisch zwischen Zentrum, Kern und Randgebieten der Literaturwissenschaft (unabhängig von nationalphilologischen Schwerpunktsetzungen) unterscheidet (siehe Schema S. 371).

Dieses 'planetarische Modell' vermeidet hierarchische Strukturen. Es möchte auf ein offenes System der Fachwissenschaft hinaus und zugleich jenen Kern festhalten, der, als Ursprungsort der für eine ganze Klasse von Fachdisziplinen zuständigen modi procedendi, jene Diskursrationalität garantiert, auf die

53 Geertz (Anm. 5) $69 \mathrm{f}$.; Leo Spitzer, Linguistics and Literary History (Princeton: Princeton UP, 1948).

54 A. van Gennep, Übergangsriten, 1909 (Frankfurt/M.: Campus, 1986). Zur imaginativ-literarischen Struktur ethnographischer Schreibweisen vgl. die aufschlußreichen buchkritischen Essays von C. Geertz, Works and Lives: The Anthropologist as Author (Stanford: Stanford UP, 1988) und den Sammelband Writing Culture: The Poetics and Politics of Ethnography, ed. J. Clifford and G. E. Marcus (Berkeley: U of California P, 1986). Scharfe Kritik an dieser Diskursmischung und ihrem impliziten Relativismus übt Ernest Gellner, Postmodernism, Reason and Religion (London: Routledge, 1992), hier bes. das Kapitel "Relativismus über Alles" (Dt. im Original). 
Interdisziplinäre

Peripherie
Medienwissenschaften

Fachliches Zentrum

Linguistik
Soziologie

Komparatistik

$$
\begin{array}{cc}
\multicolumn{2}{c}{\text { Textkritik }} \\
\text { Poetik } & \text { Textheorie }
\end{array}
$$

Ästhetik

Kerngebiet: Methodologie

Psychologie (Hermeneutik/Historik/Semiotik)

Literarhistorie

Fachgeschichte

Geschichte

Anthropologie

Theaterwissenschaft

das Gespräch inter disciplinas angewiesen ist. ${ }^{55}$ Offen ist die Entscheidung, für welche der auf der peripheren Kreisbahn angesiedelten Disziplinen man optiert, und selbstverständlich sind die Entfernungen zwischen Zentrum und Peripherie so variabel wie die Wahl der Ausgangsfragen.

Mögliche Anknüpfungspunkte sehe ich dort, wo im Sinne des Mauthner-Mottos der Kulturbegriff in "Verwirrung" gebracht wird, positiv gesprochen: in den Wissenschaften, in denen er nicht-normativ sowie funktional ausdifferenziert und temporalisiert in Erscheinung tritt. Kultursoziologie und Kulturanthropologie sind hierfür die einschlägigen Adressen; um Namen und Titel zu nennen: Pierre Bourdieus Soziologie der symbolischen Formen (zuerst 1970), Clifford Geertz' Art as a Cultural System (1976), Victor Turners The Anthropology of Performance

55 Erläuterung zum Kerngebiet: Hermeneutik = Theorie des Verstehens; Historik $=$ Theorie der hist. Erklärung; Semiotik $=$ Theorie der Zeichenanalyse. Die Akte des Verstehens, des Erklärens, der Analyse bilden eine gemeinsame methodolog. Basis der Kulturwissenschaften. Sie variieren zwar von Fach zu Fach, verlieren dadurch aber nicht ihre Familienähnlichkeit und sind insofern die eigentliche Clearingstelle für interdisziplinäre Kontakte. 
(zuerst 1987) und - um eine wenig ältere Position kultursoziologischer Literaturforschung zu vergegenwärtigen - Raymond Williams Studien Über den Prozeßcharakter von Literatur und Kultur (1961-75). ${ }^{56}$ Die Erscheinungsdaten sind hier insofern von Interesse, als sie die Lust am Konzeptionellen und Theoretischen wieder ins Gedächtnis rufen, die vor allem in den 60er und 70er Jahren fröhliche Urständ gefeiert hat.

Was die soeben beim Namen genannten, hier und da in Einzelheiten doch sehr unterschiedlichen Positionen verbindet, ist dreierlei:

- Zum einen favorisieren sie einen Analysemodus, der Kulturen zuallererst aus dem Blickwinkel der Praxis, nicht der Poiesis betrachtet. Hier sind die Einzelwerke nicht privilegierte Orte kultureller Repräsentation, sondern eher so etwas wie Einstiegsmöglichkeiten. Die Interpretationsversuche richten sich unter dieser Voraussetzung auf die in den kulturellen Einzelmilieus kodifizierten Prozesse der Formgebung, der Bedeutungsbildung sowie ihrer kreativen Durchbrechung und folgen dem Grundsatz: Die Bedeutung der Werke entsteht und verwandelt sich im Medium gesellschaftlicher Kommunikation. Konsequenterweise kennzeichnet dieser Kulturbegriff nicht eine homogene Struktur, sondern variable, durch Konflikte, Überschneidungen, Brechungen und Verwerfungen charakterisierte Systemprozesse. Übernimmt der Literaturwissenschaftler diese Perspektive, so entlastet ihn das zwar nicht von der Mühe philologischer Erkenntnis, ${ }^{57}$ er wird den Einzeltext dann aber nicht wie ein scheinbar nur aus sich selbst heraus verständliches homogenes Kunstprodukt seinem Deutungswissen assimilieren, sondern ihn als Kreuzungspunkt anderer, auch der nichtoder subliterarischen kulturellen Praktiken betrachten.

56 Geertz' Essay findet sich in dem in Anm. 5 erwähnten Sammelband. Der Williams-Titel bezieht sich auf den von H. G. Klaus hrsg. und übersetzten Sammelband Innovationen: Über den Prozeßcharakter von Literatur und Kultur (Frankfurt: Syndikat, 1977), der neben einigen Aufsätzen wichtige Auszüge aus The Long Revolution (1961) enthält.

57 In der Bedeutung der von Peter Szondi in "Über philologische Erkenntnis" diskutierten Prinzipien; Hölderlin-Studien (Frankfurt/M.: Suhrkamp, 1970) $9 \mathrm{ff}$. 
- Zum zweiten verbindet die genannten Positionen ein Interesse für das, was ich hier in zugespitzter Weise das Widerspiel von Regularität und Kontingenz im kulturellen Handeln, nicht zuletzt in dessen ästhetischen Manifestationen nennen möchte. Das Artefakt - ein Bild, eine Skulptur, ein Gedicht, eine Aufführung - als Teil eines kulturellen Systems - und sei dieses auch nur als Zeit- oder Kunststil definiert - zu betrachten, heißt: es in kollektive Zusammenhänge und die in ihnen wirksamen Konventionen einzubetten. Diese Operation ist notwendig, um das, was man in Abwandlung einer Formel Bourdieus den "Dissens im Konsens" nennen könnte, in Erfahrung zu bringen. Denn noch die ausgefallenste, will sagen: kraftvollste Abweichung einer individuellen Kunstschöpfung schließt - auch und gerade in der Geste des Widerstands - an eine gegebene kulturelle Ordnung, an mehr oder weniger verfestigte Normen und Muster an. Jedes Artefakt ist eine Ellipse. ${ }^{58}$ Was Norm, was Abweichung ist, läßt sich nur beantworten, wenn die Analyse das ganze kulturelle Feld und die in ihm geltenden Konventionen in den Blick nimmt. Nicht Kontinuität und Stetigkeit, sondern Konflikt und Übergang sind hier die adäquaten Beschreibungsbegriffe. Der Prozeß z. B. der Kanonbildung, der in jeder Kultur zu beobachten ist, verläuft in der Regel dramatisch: als Konflikt zwischen - wie Turner das nennt - "Struktur und Antistruktur". Kanon bedeutet zwar Norm und Regel, impliziert aber in symboltheoretischer Perspektive eine polare Spannung, die es erlaubt, zwischen dominanten und virtuellen, vom Kanon verdrängten, in Latenz jedoch subversiv weiterwirkenden Bedeutungen zu unterscheiden. Von daher läßt sich die Beziehung zwischen literarischem bzw. Kunst-Kanon und den anderen Normsystemen innerhalb einer Kultur neu bestimmen. Das traditonelle Kongruenzmodell, das um der Homogenität willen die ästhetische 'Hoch'-Kultur mit der Gesamtkultur zur Deckung bringen wollte, unterschlägt die in jeder Kultur wirksamen Konflikte zwischen Kanondominanz und anti-kanonischer Variabilität. 59

58 Bourdieu (Anm. 42) 116.

59 Die Erörterung einer polaren, spannungsgeladenen Symbolstruktur 
- Der dritte und letzte hier zu glossierende Punkt berührt die schwierige Frage, wie sich der Kulturwissenschaftler zu der Tatsache verhält, daß er seine historischen oder exotischen Objekte unter Voraussetzungen auswählt und interpretiert, die diesen selber fremd sind. Das von Dilthey vertretene Sicheinfühlen ist einer assimilatorischen Interpretation ähnlich, in der das Andere im Andern hinter dessen Geltungsanspruch an die Gegenwart zurücktritt. Sich unter dieser Voraussetzung das Vergangene anzueignen, entsprach einer Auffassung von kultureller Kontinuität, die, als fortschreitender Bildungsprozeß gedacht, Nähe und Ferne durch ein Band zeitübergreifender Sinnverwandtschaften miteinander verknüpfte. In der ethnologischen Kulturhermeneutik hingegen steht nicht die Aneignung, sondern die Frage nach dem Anderssein des Fremden im Vordergrund. Was sind die Konsequenzen, orientieren sich die "Geisteswissenschaften" - wofür einige Indizien sprechen - an der Kulturanthropologie als neuer Leitwissenschaft?

Betrachten wir zunächst in aller Kürze den Aspekt der Professionalisierung: Für sie ist die Frage nach jener Kompetenz entscheidend, die sich in der Anwendung rationaler Forschungsund Auslegungsstrategien bewährt. Heute erscheinen die Gesetze der Kultur- wie der Kunstrezeption als ein Spezialfall der Gesetze des Wissenserwerbs. Bourdieu verweist auf den historischen Zusammenhang zwischen der Autonomisierung der Künste und der Herausbildung autonomer Kunstwissenschaften. Wie die Warburg-Schule, an die er anknüpft, wendet er sich gegen den Mythos des "reinen Sehens", da dieser das Vertraute zur Basis eines unmittelbaren Zugangs zu einem Sonderfall macht, der als solcher nur in der Vorstellungswelt des Interpreten existiert. Bourdieus Interesse gilt der "Appropriation” kultureller Güter auf der Basis reflektierter, professionell verfeinerter, lehr- und lernbarer Entschlüsselungskompetenzen. Mit dem Hinweis auf die Rationalität des Kompetenzerwerbs und seiner methodischen Anwendung in Kultur- und

findet sich nicht nur bei Warburg (s. o.), sondern unter anderen Vorzeichen auch in den kulturanthropologischen Arbeiten von Victor Turner und Mary Douglas; vgl. zu letzteren die Darstellung Wolfgang Lipps (Anm. 18) $40 \mathrm{ff}$. 
Kunstwissenschaften sucht er das "Dogma der unbefleckten Erkenntnis" (Nietzsche) zu entzaubern, das lange genug dazu diente, die akademische Arbeit am Sinn hinter dem Schleier priesterlicher Hoheit zu verbergen.

So lehrreich und produktiv Bourdieus Kritik an den akademischen Obskurantismen ist, sie gibt auf die Frage nach der Verständigung zwischen Eigenem und Anderem keine Antwort. Vielleicht liegt eine Antwort in jenen "puzzles of translation", die Geertz mit Worten umschreibt, die an die alte semiotische Formel aliquid stat pro aliquo gemahnt; denn daß "etwas für etwas steht", legt die Assoziation nahe, es stehe "für etwas anderes". Vielleicht liegt die Antwort aber auch in der von Bourdieu beschriebenen methodischen Rationalität, akzeptiert man sie als einzige kulturübergreifende Universale der modernen Welt. ${ }^{60}$ Nicht alles ist auf einer Seite zu entscheiden.

HeIDELberG

DIETRICH HARTH

60 Die letzte These vertritt Ernest Gellner, der Widersacher von Geertz (vgl. Anm. 54). 